\title{
CareNet: An Integrated Wireless Sensor Networking Environment for Remote Healthcare
}

\author{
Shanshan Jiang, Yanchuan Cao, Sameer lyengar, Philip Kuryloski, Roozbeh Jafari \\ Yuan Xue, Ruzena Bajcsy, Stephen Wicker
}

\begin{abstract}
CareNet is an integrated wireless sensor environment for remote healthcare that uses a two-tier wireless network and an extensible software platform. CareNet provides both highly reliable and privacy-aware patient data collection, transmission and access. This paper describes our system architecture, software development, and the results of our field studies.
\end{abstract}

\section{INTRODUCTION}

The cost of health care for our nation's aging population has become a national concern. According to the U.S. Census Bureau, the number of people over the age of 65 is expected to hit 70 million by 2030, having doubled since 2000 . Health care expenditures in the United States are projected to rise to $15.9 \%$ of the GDP ( $\$ 2.6$ trillion) by 2010 .

Recent advances in wireless sensor networks have made it possible to deploy wearable sensors on the bodies of patients in a residential setting, allowing continuous monitoring of physiological signals (such as ECG, blood oxygen lev-

\begin{abstract}
* Shanshan Jiang, Yanchuan Cao and Yuan Xue are from the EECS department at Vanderbilt University, their email addresses are \{shanshan.jiang,yanchuan.cao,yuan.xue\}@vanderbilt.edu; Sammer Iyengar and Ruzena Bajcsy are from the EECS department of the University of California at Berkeley, their email addresses are \{sameer,bajcsy\}@eecs.berkeley.edu; Philip Kuryloski and Stephen Wicker are from the ECE department at Cornell University, their email addresses are pjk25@cornell.edu and wicker@ece.cornell.edu. Roozbeh Jafari is from the department of Electrical Engineering of the University of Texas at Dallas, his email address is rjafari@utdallas.edu. This work was supported in part by TRUST (The Team for Research in Ubiquitous Secure Technology), which receives support from the National Science Foundation (NSF award number CCF-0424422) and the following organizations: AFOSR (\#FA9550-06-1-0244), Cisco, British Telecom, ESCHER, HP, IBM, iCAST, Intel, Microsoft, ORNL, Pirelli, Qualcomm, Sun, Symantec, Telecom Italia and United Technologies.
\end{abstract}

Permission to make digital or hard copies of all or part of this work for personal or classroom use is granted without fee provided that copies are not made or distributed for profit or commercial advantage and that copies bear this notice and the full citation on the first page. To copy otherwise, to republish, to post on servers or to redistribute to lists, requires prior specific permission and/or a fee.

BodyNets 2008, March 13-15 Tempe, Arizona, USA

Copyright @ 2008 ICST 978-963-9799-17-2

DOI 10.4108/ICST.BODYNETS2008.2965 els) and other health related information (such as physical activity levels). The application of wireless sensor networks in a medical care environment provides a unique opportunity to shift health care outside a traditional clinical setting to a patient/home-centered setting, and to reduce healthcare expenses through more efficient use of clinical resources and earlier detection of medical conditions.

There remains a significant gap between the availability of the sensing technology and our ability to bring it into general use for home medical sensing. A medical sensing system must provide reliable and privacy-preserving information transmission between patients' homes and the care giver. In light of such need, we present CareNet, an integrated wireless sensor environment for remote healthcare. Our system has the following features.

- High reliability and performance. Our wireless networking infrastructure features a two-tier design. A high-performance IEEE 802.11 wireless network is used as the backbone structure to provide local area communication coverage, while the wearable body sensors communicate with the base-stations of the backbone structure directly. Compared with sensor networks in which wireless communications are solely based on IEEE 802.15.4 standard, this design greatly improves system reliability and performance. Further, the backbone structure can also be equipped with audio and video sensors which support additional multimedia communication needs in the homecare services.

- Good scalability and extensibility. Our networking and security software at the backbone network is built on top of the ACE [1] environment, which is commonly used to build extensible concurrent and networking applications. Using a backbone structure, our hybrid network design scales much better than a pure IEEE 802.15.4-based sensor network.

- Privacy aware data confidentiality protection. Privacy and data confidentiality is a primary consideration in our system design. Our system design features built-in secure communication components which are adaptively implemented for different networking environments and used at all communication phases of the system.

- Integration with web-based patient portal. In our system, the sensor data collection does not mark the end, but simply the beginning of data transport. Data from the patient centric sensor network will be collected in a web-based patient portal that is under development at Vanderbilt Medical School. The portal allows care givers to efficiently access sensor network data through a unified medical record system. The patient portal also allows for arbitration of access permissions for different parties (e.g. families, physicians, 
nurses, and insurance companies).

In this paper, we present the design and system prototype of one remote healthcare service - physical activity monitoring, which is built on top of this environment. The physical activity monitoring service is able to provide continuous physical movement monitoring through sensors that are equipped with accelerometers and gyroscopes, as well as fall detection and associated alarms. The system is also equipped with a video sensor network which provides ondemand video information to verify the physical activity monitoring results.

Collaborating with Vanderbilt Homecare Services, we have deployed our system at four patients' homes, and tested our system over a 24 -hour period. This paper reports the performance of our system prototype and presents preliminary experimental results.

The remainder of this paper is organized as follows. Section 2 describes our network architecture and hardware platform. Section 3 presents the software design and prototype. We report our experiment study and results in Section 4 and conclude the paper in Section 5.

\section{SYSTEM ARCHITECTURE}

CareNet is built upon a heterogeneous networking infrastructure which involves the patient data collection, transmission, and access phases, as shown in Figure 1. This paper focuses its discussion on the networking and system design of the data collection phase.

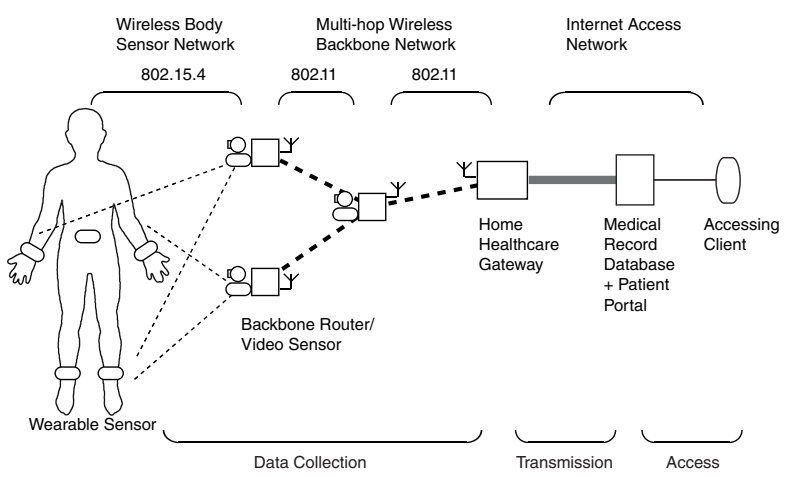

Figure 1: System Architecture

As illustrated in the figure, a two-tier wireless network is used to provide data sensing, collection, transmission and processing functions. At the lower tier, a body sensor network consisting of lightweight wearable sensors provides data sensing and transmission functions. These sensors can communicate with the base-station sensors (which are attached to the backbone wireless network) directly using IEEE 802.15.4 wireless standard. We use Telos motes as the hardware devices. For movement sensing and fall detection, these motes are equipped with accelerometers and gyroscopes. At this tier, sensor devices are lightweight, wearable and mobile, which also means they have low computation, communication power and small amount of memory. So in our design, only necessary computational and communication tasks are implemented at these devices.

At the upper tier of the network is a multi-hop IEEE 802.11-based wireless network which provides a high-performance backbone structure for packet routing. We use Stargate sin- gle board computers as the hardware devices. The backbone routers are connected to the base-station motes which communicate with the mobile wearable sensors directly. The Stargate board can also be connected with a web camera and serve as a video sensor. Equipped with IEEE 802.11 wireless adaptors, the backbone routers communicate with each other and relay the movement sensing data as well as video streams to the home healthcare gateway. Using IEEE 802.11 wireless communication standard, this stationary backbone structure provides a high-performance and high-reliability packet routing service. Since IEEE 802.11 has a larger communication range than IEEE 802.15.4, our design also scales much better in terms of local area communication coverage. Finally, the home healthcare gateway serves as an interface between the patient's home and the caregiver's medical system, which processes all the sensing data and transmits them to the remote medical care system.

\section{SOFTWARE DESIGN}

CareNet is also built upon a multi-layered software infrastructure based on the features and functions at each of the network tiers. The overall software architecture is shown in Figure 2.
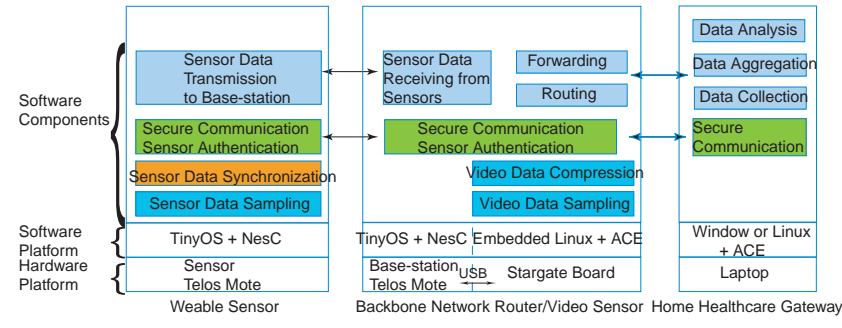

Figure 2: Software Architecture

We use the TinyOS [2] operating system and the NesC [3] programming language to implement the movement data sensing at the wearable sensors and the data transmission between the mobile wearable sensors and the base-stations. The major functions of the wearable sensors are to sample, synchronize (with the base-stations), and transmit the movement data. Beacon messages are used for hand-offs between the base-stations and the mobile sensors when the mobile sensors are moving into or out of the communication range of the base-station sensors. A customer-designed and -implemented protocol based on TinySec [4] is used for mobile sensor authentication and secure communication between mobile sensors and the base-stations.

We use Embedded Linux operating system and the ACE [1] programming environment to implement the network communication among the backbone network routers and between the backbone routers and the home healthcare gateway. ACE is open-source software based on $\mathrm{C}++$, which encapsulates OS concurrency and network programming APIs. We take advantage of ACE's strong communication and concurrency capabilities in our implementation. There are two major functions implemented at this layer: as backbone routing structure, software components are built to route and forward the video and sensor data to the home healthcare gateway; as video sensors, video data sampling and compression functions are also implemented. There are two major design considerations in the backbone network rout- 
ing infrastructure:

- Application-level routing. We implement the routing protocol among the backbone routers at the application level using TCP sockets. The reason behind this design choice is that it can easily deal with the data loss and data replication in the wireless transmission. We implement a multihop packet forwarding mechanism through TCP streams in ACE. Since the backbone network is stationary most of the time, we use a semi-static routing protocol to forward packets based on a routing table that can be either preconfigured manually or updated by HELLO messages every hour. The routing table selects the IP address and port number of the next hop node by searching the destination node inside the packet header. A backbone router may need to forward the data streams from more than one video or movement sensor; the data streams will be forwarded simultaneously through different threads in the system. Using TCP, the secure communication among backbone routers is implemented based on SSL.

- Mobile sensor hand-off. To ensure reliable packet delivery during the mobile sensor and base-station hand-offs, packets from the mobile sensors will be received by all basestations within their transmission ranges. This means that the same data may be forwarded through more than one base-station (as well as backbone routers). To remove the duplicate data packets from the backbone network, each sensor data packet is marked with a timestamp in its packet header. Duplicate data packets that arrive late at the queue of a router will be dropped. The remaining duplicate and out-of-order packets will be dropped and sorted at the home healthcare gateway.

We use the Linux operating system and the ACE programming environment to develop the application software for the home healthcare gateway, whose function is to collect, aggregate, analyze, and forward the video and movement sensor data.

\section{EXPERIMENT}

Collaborating with Vanderbilt Homecare Services, we identified four senior volunteers to participate the experiment of our system prototype. In our experiment, five sensor motes are mounted on each volunteer, two on the wrists, two on the ankles, and one on the waist. Each sensor mote is capable of recording accelerations in three dimensions $(x, y$, and $\mathrm{z}$ axis) as well as gyroscope in two dimensions ( $\mathrm{x}$ and $\mathrm{y}$ axis).

The experiment process involves a controlled experiment, where volunteers are required to perform a set of designed movements, and an uncontrolled experiment, where volunteers can perform their daily physical activities. The designed movement set includes (1) Vertical stretching of each arm; (2) Vertical stretching of both arms; (3) Drinking water; (4) Sit-to-stand and stand-to-sit; (5) Raising each leg; (6) Raising both legs. Each set of movements is repeated 5 times in the experiment. While sensor motes are recording the movement data, one video camera is recording the volunteer's motion images simultaneously. Both sensor movement data and video images are sent back and stored in the home healthcare gateway. We synthesized movement and video data based on their timestamps, then playback and compare their synchronicity.

Figure 3 shows the result of "sit-to-stand and stand-tosit" experiment. The sensor motes communicated on a sin- gle channel. The sensor data rate is about $33 \mathrm{~Hz}$. (Each mote sent a packet every $30 \mathrm{~ms}$. With five motes, the sensor network transmitted a packet every $6 \mathrm{~ms}$ on average). We received more than 400 movement packets from each of the five sensors, and for each packet we recorded the $\mathrm{X}-$, $\mathrm{Y}$ , Z-axis accelerations and the rotations around $\mathrm{X}-$, Y-axis. We also show the corresponding image data when receiving movement packets 100 and 140 . The image data are used for movement data verification and analysis.

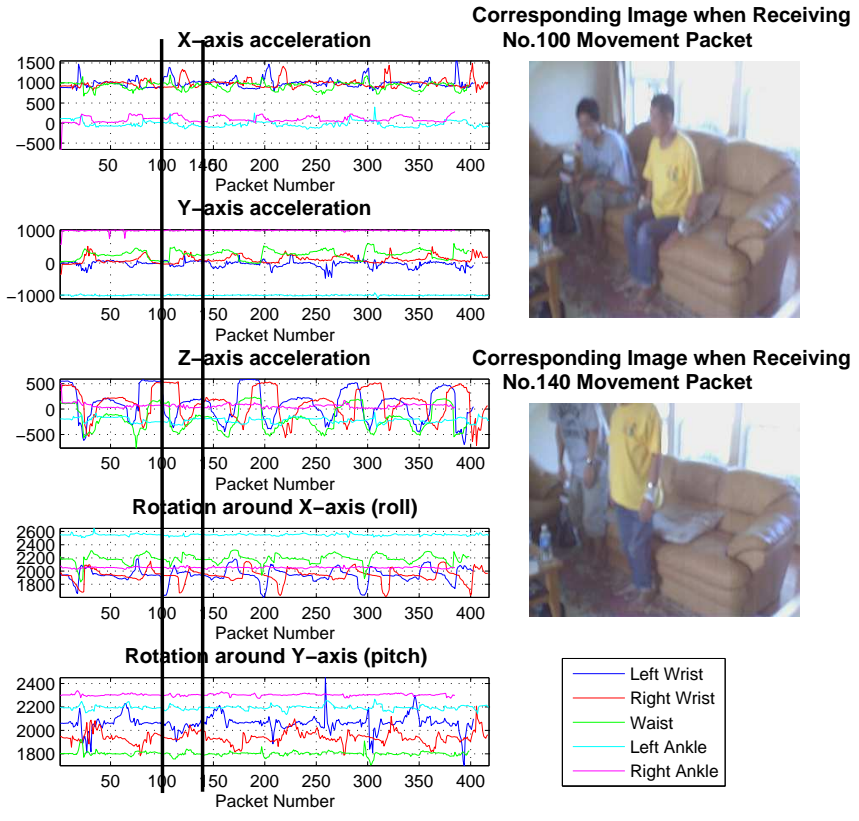

Figure 3: Sit-to-stand and stand-to-sit experiment

\section{CONCLUDING REMARKS}

This paper presents CareNet, an integrated wireless sensor environment for remote healthcare. The proposed system features a two-tier wireless network architecture, an extensible software platform, and a built-in security communication component, which effectively address the need of reliable and privacy-preserving information transmission between patients' homes and the caregiver. Our system prototype is tested at four patients' homes. The preliminary experiments show promising results for our solution.

\section{REFERENCES}

[1] http://www.cs.wustl.edu/ schmidt/ACE.html.

[2] http://www.tinyos.net/tinyos-2.x/doc/.

[3] http://nescc.sourceforge.net/.

[4] C. Karlof, N. Sastry, and D. Wagner, "TinySec: A Link Layer Security Architecture for Wireless Sensor Networks," in Proc. of HCMDSS Workshop, 2005. 